Faculty of Mathematical Sciences

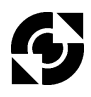

University of Twente

The Netherlands
P.O. Box 217

7500 AE Enschede

The Netherlands

Phone: +31-53-4893400

Fax: +31-53-4893114

Email: memo@math.utwente.nl

www.math.utwente.nl/publications

Memorandum No. 1628

Semiproportional values for TU games

A.B. Khmelnitskaya ${ }^{1}$ And T.S.H. Driessen

JunE, 2002

ISSN 0169-2690

\footnotetext{
${ }^{1} \mathrm{SPb}$ Institute for Economics and Mathematics, Russian Academy of Sciences, 1 Tchaikovsky St., 191187 St. Petersburg, Russia
} 


\title{
Semiproportional Values for TU Games
}

\author{
Anna B. Khmelnitskaya ${ }^{1}$ and Theo S.H. Driessen ${ }^{2 \star \star}$ \\ $1 \mathrm{SPb}$ Institute for Economics and Mathematics Russian Academy of Sciences, \\ 1 Tchaikovsky St., 191187 St.Petersburg, Russia, \\ e-mail: anna@AK3141.spb.edu \\ 2 Faculty of Mathematical Sciences, University of Twente, \\ P.O. Box 217, 7500 AE Enschede, The Netherlands, \\ e-mail: t.s.h.driessen@math.utwente.nl
}

\begin{abstract}
The goal of the paper is to introduce a family of values for transferable utility cooperative games that are proportional for twoperson games and as well satisfying some combinatorial structure composed by contributions of complementary coalitions or, to less extent, marginal contributions by players.
\end{abstract}

Keywords: cooperative TU game, value, proportional sharing, probabilistic model

Mathematics Subject Classification 2000: $91 \mathrm{~A} 12$

\footnotetext{
* This is a revised and improved version of Khmelnitskaya, A.B., and T.S.H. Driessen: "Semiproportional values for TU games," Working paper No. 10/01, International Center for Economic Research ICER, Turin, Italy, 2001. The research was done in line with research cooperation between Russian Federation and The Netherlands approved by The Netherlands Organization for Scientific Research (NWO) (Research grant corr.nr. 2000/4/ib, dos.nr. 047-008-010); it was initiated while the first author visited the Faculty of Mathematical Sciences, University of Twente, Enschede, The Netherlands (November-December 1999), and funding from the SWON (Stichting Wiskunde Onderzoek Nederland) is also sincerely appreciated. The paper was partially written during her visit to International Center for Economic Research (ICER) at Turin, Italy, (February-May 2001) whose hospitality and financial support are gratefully acknowledged as well.

** We would like to thank Natalia Naumova and Elena Yanovskaya for interesting remarks and comments.
} 


\section{Introduction}

In the framework of the solution theory for two-person games there are two basic rules, namely the standard solution and the proportional sharing of the surplus. In both, each player first gets his own guaranteed payoff and then the remaining surplus or overall savings are shared either equally or proportionally to players' initial contributions. Most known values, in particular, the Shapley value [10] and the Banzhaf value [1], are standard for two-person games. Values that appear to be proportional for two-person games are studied less. Among them it is worth mentioning the proper Shapley value of Vorob'ev-Liapunov [11], the proportional value introduced independently by Ortmann [8] and Feldman [4], and the weighted entropy solution of Naumova [7]. The goal of this paper is to introduce a family of values that are proportional for two-person games and as well satisfying some combinatorial structure composed by contributions of complementary coalitions or, to less extent, marginal contributions by players.

Sect. 2 introduces basic definitions and notation. In Sect. 3, we define a family of the semiproportional values. The payoff to a player in a game due to the semiproportional value is a weighted sum of relative gains achieved by coalitions containing the player. For any such coalition, its relative gain in comparison to the complementary coalition is determined by the proportionality rule applied to the two-person subgame in which the coalition and its complementary one are involved as two individuals in order to divide the overall savings. We present conditions on the collection of weights that guarantee the semiproportional value to be proportional for two-person games, efficient, proportionally marginalist, and probabilistic. We also consider some examples of semiproportional values. In Sect. 4 we show that the semiproportional values can be supported by a bilateral probabilistic model. The player set is randomly partitioned into two nonempty complementary coalitions and from each of them a "leader" or "representative" is chosen. The two leaders split the total resources available to all the players playing some chosen two-person game and having in mind the worths of the two coalitions involved, and then each one of them pays, out of his share, a wage to every member of his coalition as specified by some a priori given wage vector, and the rest keeps himself. A payoff vector is considered to be consistent with respect to the probabilistic model if it coincides with the expected payoff. We show that for every non-negative game with nonzero sum of worths of any two complementary coalitions, there exists a unique efficient payoff vector consistent with the probabilistic model under the proportional solution for two-person games and it is given by a certain semiproportional value dependent on the probability distributions underlying the probabilistic model. Because of this feature we choose the name "semiproportional" for the introduced family of values. We also show that there exists the only one symmetric semiproportional value presenting the unique efficient payoff vector consistent with the relevant probabilistic model under the proportional solution for two-person games, and it coincides with the symmetric and proportionally marginalist Shapley type semiproportional value. For the Shapley type semiproportional value we provide an axiomatic characterization by efficiency, symmetry, and proportional marginalism, similar to that 
of Young [13] for the Shapley value by efficiency, symmetry, and marginalism. The proofs are given in Appendix.

\section{Preliminaries}

A cooperative game with transferable utility (TU game) is a pair $\langle N, v\rangle$, where $N=\{1, \ldots, n\}$ is a finite set of $n \geq 2$ players and $v: 2^{N} \rightarrow \mathbb{R}$ is a characteristic function, defined on the power set of $N$, satisfying $v(\emptyset)=0$. A subset $S \subseteq N$ (or $S \in 2^{N}$ ) of $s$ players is called a coalition, and the associated real number $v(S)$ presents the worth of the coalition $S$. For simplicity of notation and if no ambiguity appears, we shall write $v$ instead of $\langle N, v\rangle$ when refer to a game. We also omit the braces when writing one or two-player coalitions such as $\{i\}$ or $\{i, j\}$. The set of all games with a fixed player set $N$ we denote $\mathcal{G}_{N}$. In what follows we consider only single-valued solutions usually called values. For any set of games $\mathcal{G} \subseteq \mathcal{G}_{N}$, a value on $\mathcal{G}$ is a mapping $\xi: \mathcal{G} \rightarrow \mathbb{R}^{n}$ that associates with each game $v \in \overline{\mathcal{G}}$ a vector $\xi(v) \in \mathbb{R}^{n}$, where the real number $\xi_{i}(v)$ represents the payoff to player $i$ in the game $v$.

A value $\xi$ is efficient if, for all $v \in \mathcal{G}$,

$$
\sum_{i \in N} \xi_{i}(v)=v(N)
$$

A value $\xi$ is symmetric if, for all $v \in \mathcal{G}$, for any permutation $\pi: N \rightarrow N$, and for all $i \in N$,

$$
\xi_{\pi(i)}\left(v^{\pi}\right)=\xi_{i}(v),
$$

where $v^{\pi}(S)=v(\pi(S))$ for all $S \subseteq N, S \neq \emptyset$.

A value $\xi$ is marginalist if, for all $v \in \mathcal{G}$, for every $i \in N, \xi_{i}(v)$ depends only upon the $i$ th marginal utility vector $\{v(S \cup i)-v(S)\}_{S \subseteq N \backslash i}$, i.e.,

$$
\xi_{i}(v)=\phi_{i}\left(\{v(S \cup i)-v(S)\}_{S \subseteq N \backslash i}\right),
$$

where $\phi_{i}: \mathbb{R}^{2^{n-1}} \rightarrow \mathbb{R}^{1}$.

A player $i$ is a null-player in the game $v \in \mathcal{G}$ if $v(S \cup i)=v(S)$, for every $S \subseteq N \backslash i$. A value $\xi$ possesses the null-player property if, for all $v \in \mathcal{G}$, for every null-player $i$ in $v, \xi_{i}(v)=0$.

Remark 2.1. Under marginalism, the null-player property is equivalent to all functions $\phi_{i}, i \in N$, in the definition of marginalism (1), at $\mathbf{0} \in \mathbb{R}^{2^{n-1}}$ being equal to 0 , i.e., $\phi_{i}(\mathbf{0})=0$, for every $i \in N$.

For every vector $\left(x_{i}\right)_{i \in N} \in \mathbb{R}^{n}$ and every coalition $S \subseteq N, S \neq \emptyset$, we write $x(S):=\sum_{i \in S} x_{i}$.

In the framework of the solution theory for two-person games, we recall two basic rules, namely the standard solution and the proportional sharing of the surplus, when each player first gets his own guaranteed payoff and then the remaining surplus or overall savings are shared either equally or proportionally 
to players' initial contributions. A value $\xi$ is standard for two-person games if, for all $v \in \mathcal{G}_{\{1,2\}}$, for every $i=1,2$, the surplus of individual worths is divided equally among both players, that is

$$
\xi_{i}(\langle\{1,2\}, v\rangle)=v(i)+\frac{v(1,2)-v(1)-v(2)}{2} .
$$

A value $\xi$ is proportional for two-person games if, for any $v \in \mathcal{G}_{\{1,2\}}$ such that $v(1)+v(2) \neq 0$, for every $i=1,2$, the surplus is distributed proportionally to the individual worths, that is

$$
\xi_{i}(\langle\{1,2\}, v\rangle)=v(i)+\frac{v(i)}{v(1)+v(2)}[v(1,2)-v(1)-v(2)],
$$

or equivalently

$$
\xi_{i}(\langle\{1,2\}, v\rangle)=\frac{v(i)}{v(1)+v(2)} v(1,2) .
$$

Our aim is to introduce a family of values that are proportional for two-person games and satisfy some combinatorial structure composed by contributions of complementary coalitions or, to less extent, marginal contributions by players.

Throughout the remainder of the paper, it is tacitly assumed that we consider non-negative games of the class

$$
\mathcal{G}_{N}^{+}=\left\{v \in \mathcal{G}_{N} \mid v(S) \geq 0, v(S)+v(N \backslash S) \neq 0, \quad \text { for all } S \subseteq N\right\} .
$$

For the class of positive games we use notation $\mathcal{G}_{N}^{++}$,

$$
\mathcal{G}_{N}^{++}=\left\{v \in \mathcal{G}_{N} \mid v(S)>0, \quad \text { for all } S \subseteq N, S \neq \emptyset\right\} .
$$

Obviously, $\mathcal{G}_{N}^{++} \subset \mathcal{G}_{N}^{+}$.

With every game $v \in \mathcal{G}_{N}^{+}$we associate its so-called constant-sum normalization $v^{c} \in \mathcal{G}_{N}^{+}$defined to be

$$
v^{c}(S)=\frac{v(S)}{v(S)+v(N \backslash S)} v(N), \quad \text { for all } S \subseteq N .
$$

Observe that, for all games $v \in \mathcal{G}_{N}^{+}$

(i) $v^{c}$ is a constant-sum game, i.e.,

$$
v^{c}(S)+v^{c}(N \backslash S)=v^{c}(N)=v(N), \quad \text { for all } S \subseteq N ;
$$

(ii) $v^{c}$ is invariant under $c$-normalization, i.e.,

$$
\left(v^{c}\right)^{c}(S)=v^{c}(S), \quad \text { for all } S \subseteq N ;
$$

note that $v^{c}=v$ whenever the initial game $v$ is a constant-sum game itself (e.g., for additive games); 
(iii) the core of the $c$-normalization of a two-person game consists of a unique payoff vector, which agrees with the proportional solution for the two-person game;

(iv) for any coalition $S \subseteq N$ and its complement $N \backslash S$, the proportionality rule (3) applied to their two-person subgame of the initial game agrees with the standard solution (2) applied to their two-person subgame of its constantsum normalization $v^{c}$, i.e.,

$$
\frac{v(S)}{v(S)+v(N \backslash S)} v(N)=v^{c}(S)+\frac{v^{c}(N)-v^{c}(S)-v^{c}(N \backslash S)}{2} .
$$

For every coalition $S \subseteq N$ the amount $v^{c}(S)$ has a natural interpretation. Indeed, the worth $v(S)$ is the gain that the coalition $S$ can guarantee to obtain itself. But cooperating with other players of $N$ and sharing together with them the total gain of the amount $v(N)$ the members of $S$ may expect their share to be equal to $v^{c}(S)$ which for instance in case of superadditive game certainly not worse than their self-evaluation $v(S)$. By the similar way, for any game $v \in \mathcal{G}_{N}^{+}$, for every player $i \in N$ and any coalition $S \subseteq N \backslash i$, the amount

$$
v^{c}(S \cup i)-v^{c}(S)=\frac{v(S \cup i)}{v(S \cup i)+v((N \backslash S) \backslash i)} v(N)-\frac{v(S)}{v(S)+v(N \backslash S)} v(N)
$$

is reasonable to consider as the proportional marginal utility of the player $i$ to the coalition $S$.

A value $\xi$ defined on the class of games $v \in \mathcal{G}_{N}^{+}$is proportionally marginalist if for all $v \in \mathcal{G}_{N}^{+}$, for every $i \in N, \xi_{i}(v)$ depends only on $i$-th vector of proportional marginal utilities $\left\{v^{c}(S \cup i)-v^{c}(S)\right\}_{S \subseteq N \backslash i}$, i.e.,

$$
\xi_{i}(v)=\phi_{i}\left(\left\{v^{c}(S \cup i)-v^{c}(S)\right\}_{S \subseteq N \backslash i}\right),
$$

where $\phi_{i}: \mathbb{R}^{2^{n-1}} \rightarrow \mathbb{R}^{1}$.

Remark 2.2. Notice that every proportionally marginalist value depends only on

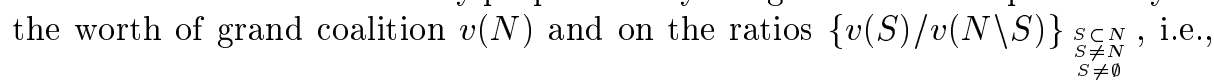
on the relative worth of all complementary nonempty coalitions, and it can be reduced to a marginalist value defined on $\mathcal{G}_{N}^{+c}=\left\{v \in \mathcal{G}_{N}^{+} \mid v=v^{c}\right\}$.

Remark 2.3. One can easily see that the class of games $\mathcal{G}_{N}^{+c}$ coincide with the class of nonnegative constant-sum games, i.e.,

$$
\mathcal{G}_{N}^{+c}=\left\{v \in \mathcal{G}_{N} \mid v(N) \neq 0, v(S) \geq 0, v(S)+v(N \backslash S)=v(N), \text { for all } S \subseteq N\right\} .
$$

\section{Family of semiproportional values}

Definition 3.1. Let $\mathcal{W}_{N}=\left\{\gamma_{N}(S, i)\right\}_{\substack{S \subseteq N \\ i \in \mathcal{S}}}$ be a collection of weights; a priori we do not assume that all weights are non-negative. The semiproportional value 
of the game $v \in \mathcal{G}_{N}^{+}$is given by

$$
\operatorname{SPr}_{i}^{\mathcal{W}}(v)=\sum_{\substack{S \subset N \\ S \ni i}} \gamma_{N}(S ; i) \frac{v(S)-v(N \backslash S)}{v(S)+v(N \backslash S)} v(N), \quad \text { for all } i \in N
$$

or equivalently by

$$
\operatorname{SPr}_{i}^{\mathcal{W}}(v)=\sum_{\substack{S \subset N \\ S \ni i}} \gamma_{N}(S ; i)\left[v^{c}(S)-v^{c}(N \backslash S)\right], \quad \text { for all } i \in N .
$$

The payoff to a player in a game due to the semiproportional value is a weighted sum of relative gains (or losses, if $v(S)-v(N \backslash S)<0$ ) achieved by coalitions $S$ containing the player. For any such coalition $S$, its relative gain (in comparison to the complementary coalition $N \backslash S$ ) is determined by the proportionality rule applied to the two-person subgame in which the coalition and its complementary coalition are involved as two individuals in order to divide the overall savings of the amount $v(N)$. Notice that the semiproportional value reduces to the egalitarian division rule whenever all weights are zero, except for $\gamma_{N}(N, i)=\frac{1}{n}$, for all $i \in N$.

In what follows, by $e$ we denote a unitary simple game in which all nonempty coalitions are winning, i.e., $e(S)=1$, for all $S \subseteq N, S \neq \emptyset$.

Remark 3.2. Notice, that for every $i \in N$, the weight $\gamma_{N}(N, i)$ coincides with the semiproportional payoff to the player $i$ in the game $e$, i.e., for all $i \in N$, $\gamma_{N}(N, i)=\operatorname{SPr}_{i}^{\mathcal{W}}(e)$.

If the weights underlying any player's payoff represent a probability distribution for the collection of coalitions containing the player, i.e., for all $S \subseteq N$, $i \in S, \gamma_{N}(S, i) \geq 0$, and $\sum_{\substack{S \subset N, S \ni i}} \gamma_{N}(S, i)=1$, for all $i \in N$, then the semiproportional value is probabilistic. It is worth noting that, for any game $v \in \mathcal{G}_{N}^{+}$, every probabilistic proportionally marginalist semiproportional value appears to be a probabilistic value [12] with respect to the constant-sum normalization game $v^{c}$.

Now we present some conditions on the collection of weights $\mathcal{W}_{N}$ to guarantee that the semiproportional value is proportional for two-person games, efficient, or proportionally marginalist. The first statement gives a necessary and sufficient condition for the $S \operatorname{Pr}^{\mathcal{W}}$-value to be proportional for two-person games.

Proposition 3.3. The semiproportional value is proportional for two-person games if and only if the collection $\mathcal{W}_{\{1,2\}}$ of weights satisfies

$$
\gamma_{\{1,2\}}(\{i\}, i)=\gamma_{\{1,2\}}(\{1,2\}, i)=\frac{1}{2}, \quad \text { for every } i=1,2 .
$$

The next two propositions present sufficient conditions on the collection of weights $\mathcal{W}_{N}$ that provide the relevant semiproportional value to be efficient or proportionally marginalist respectively. 
Proposition 3.4. If the collection $\mathcal{W}_{N}$ of weights satisfies

$$
\sum_{i \in N} \gamma_{N}(N, i)=1
$$

and, for all $S \subset N, S \neq N, S \neq \emptyset$,

$$
\sum_{i \in S} \gamma_{N}(S, i)=\sum_{i \in N \backslash S} \gamma_{N}(N \backslash S, i),
$$

then the semiproportional value is efficient.

Proposition 3.5. If the collection $\mathcal{W}_{N}$ of weights, for every $i \in N$ and for all $S \subseteq N$ such that $S \ni i$, satisfies

$$
\gamma_{N}(S, i)=\gamma_{N}((N \backslash S) \cup i, i),
$$

then the semiproportional value is a proportionally marginalist value of the form

$$
\operatorname{SPr}_{i}^{\mathcal{W}}(v)=\sum_{S \subseteq N \backslash i} \gamma_{N}(S \cup i, i)\left[v^{c}(S \cup i)-v^{c}(S)\right], \quad \text { for all } i \in N .
$$

Remark 3.6. It is worth noting that every proportionally marginalist semiproportional value possesses the null-player property since, for every null-player $i$, for all coalitions $S \subseteq N \backslash i, v(S \cup i)=v(S)$ and $v(N \backslash S)=v((N \backslash S) \backslash i)$.

Further we discuss some examples to support the introduced family of values.

Example 3.7. Consider the setting of Definition 3.1. Then the following statements hold:

(i) If $\gamma_{N}(S, i)=\frac{(s-1) !(n-s) !}{n !}$, for all $S \subseteq N$ and all $i \in S$, then the semiproportional value is efficient (by Proposition 3.4), proportionally marginalist (by Proposition 3.5), and probabilistic with respect to constant-sum normalization as well. Furthermore, under the constant-sum normalization, the semiproportional value agrees with the Shapley value in that $\operatorname{SPr}^{\mathcal{W}}(v)=$ $S h\left(v^{c}\right)$, for all $v \in \mathcal{G}_{N}^{+}$(due to the marginalist form as given by (9) or directly from (5) if apply the alternative presentation of Rothblum [9] for the Shapley value). Therefore, in what follows we call this value the Shapley type semiproportional value and use notation $\operatorname{SPr}^{S h}(v)$. Notice that the relationship $S \operatorname{Pr}^{S h}(v)=\frac{\left.S h\left(v^{c}\right)^{2}\right)}{v(N)}$ holds as well since $v^{c}(S)-v^{c}(N \backslash S)=$ $\frac{\left(v^{c}(S)\right)^{2}-\left(v^{c}(N \backslash S)\right)^{2}}{v(N)}$, for all $S \subseteq N$. Moreover, this value can be characterized as a solution of a certain optimization problem which is related to the least square technique.

Proposition 3.8. The Shapley type semiproportional value of the game $v \in \mathcal{G}_{N}^{+}$, $S \operatorname{Pr}^{S h}(v)$, solves the following optimization problem:

$$
\begin{aligned}
& \text { minimize } \sum_{\substack{S \subset N \\
S \neq N \\
s \neq 0 \\
s \neq \emptyset}}(s-1) !(n-1-s) !\left[\frac{\frac{x(S)}{v(S)}-\frac{x(N \backslash S)}{v(N \backslash S)}}{\frac{1}{v(S)}+\frac{1}{v(N \backslash S)}}\right]^{2} \\
& \text { subject to }\left(x_{i}\right)_{i \in N} \in \mathbb{R}^{n} \text { with } x(N)=v(N) .
\end{aligned}
$$


Next consider how the Shapley type semiproportional value behaves on some known classes of games.

(a) The $S P r^{S h}$-value applied to the subclass of additive games

$$
\mathcal{G}_{a}^{+}=\left\{v \in \mathcal{G}_{N}^{+} \mid v(S)=\sum_{i \in S} v(i), \quad \text { for all } S \subseteq N\right\}
$$

gives a natural outcome, i.e., for all $v \in \mathcal{G}_{a}^{+}$and $i \in N, \operatorname{SPr}_{i}^{S h}(v)=v(i)$.

(b) On the subclass of semiadditive games

$$
\mathcal{G}_{s a}^{+}=\left\{v \in \mathcal{G}_{N}^{+} \mid v(S)=\sum_{i \in S} b(i)+\alpha, \quad \text { for all } S \subseteq N, S \neq \emptyset\right\},
$$

where $\alpha \geq 0$ and $b(i) \geq 0, i \in N$, are fixed constants, for all $v \in \mathcal{G}_{s a}^{+}$ and for every $i \in N, S P r_{i}^{S h}(v)=v(N) \frac{d(i)}{d(N)}$, with $d(i)=b(i)+\frac{2 \alpha}{n}$ and $d(N)=v(N)+\alpha$ (it is easy to check that $d(N)=\sum_{i \in N} d(i)$ ), i.e., the payoff to the player $i$ is a share of total gain $v(N)$ in proportion to the amount of $b(i)+\frac{2 \alpha}{n}$. Notice, that $d(i)$ differs from the $i$ th player's Shapley value $S h_{i}(v)=b(i)+\frac{\alpha}{n}$.

(c) For any big boss game (in particular, the landlord game) in which every coalition of players non-containing the big boss is worth nothing while all coalitions including him have positive worth, the semiproportional value allocates the total amount of $v(N)$ to the unique big boss and the other people get nothing. Indeed, for every such game its constant sum normalization $v^{c}$ becomes $v^{c}(S)=0$ if the big boss is not within the coalition $S$, and $v^{c}(S)=v(N)$ if the big boss is a member of $S$. The Shapley value of $v^{c}$ assigns everything of $v(N)$ to the big boss.

(ii) If $\gamma_{N}(S, i)=\left(\frac{1}{2}\right)^{n-1}$, for all $S \subseteq N$ and all $i \in S$, then the semiproportional value is proportionally marginalist (by Proposition 3.5), probabilistic with respect to constant-sum normalization, but not efficient. Further, under the constant-sum normalization, the semiproportional value agrees with the Banzhaf value in that $\operatorname{SPr}^{\mathcal{W}}(v)=B a\left(v^{c}\right)$, for all $v \in \mathcal{G}_{N}^{+}$(due to its marginalist form as given by (9)). In what follows we call this value the Banzhaf type semiproportional value and use notation $\operatorname{SPr}^{B a}(v)$. Similarly, the alternative relationship $\operatorname{SPr}^{B a}(v)=\frac{B a\left(N,\left(v^{c}\right)^{2}\right)}{v(N)}$ holds.

(iii) If $\gamma_{N}(N, i)=\frac{1}{n}$, for all $i \in N$, and $\gamma_{N}(S, i)=\frac{c_{n}}{s}$, for all $S \subset N, S \neq N$, and all $i \in S$, then the semiproportional value is efficient (by Proposition 3.4), and moreover, probabilistic if and only if $c_{n}=\frac{n-1}{2^{n}-2}$.

With every positive game $v \in \mathcal{G}_{N}^{++}$there is associated its inverse game (with respect to multiplication) given by the game $\frac{1}{v} \in \mathcal{G}_{N}^{++}$(since $v(S) \cdot \frac{1}{v}(S)=1$ for all $S \subseteq N, S \neq \emptyset$ ). For positive games $v$ with unitary overall savings $v(N)=1$, it turns out that the semiproportional values of a player in both games are interrelated to each other through a constant sum per player, or more precisely, the deviation of each of these two values of player $i$, measured with reference to 
the semiproportional payoff $S \operatorname{Pr}_{i}^{\mathcal{W}}(e)$ to the player $i$ in the unitary simple game $e$, is the same.

Proposition 3.9. For all positive games $v \in \mathcal{G}_{N}^{++}$and every $i \in N$, the semiproportional value satisfies

$$
\operatorname{SPr}_{i}^{\mathcal{W}}\left(\frac{1}{v}\right)+\frac{\operatorname{SPr}_{i}^{\mathcal{W}}(v)}{(v(N))^{2}}=2 \frac{\operatorname{SPr}_{i}^{\mathcal{W}}(e)}{v(N)}
$$

and so, for all positive $v \in \mathcal{G}_{N}^{++}$with $v(N)=1$, for every $i \in N$,

$$
\frac{\operatorname{SPr}_{i}^{\mathcal{W}}\left(\frac{1}{v}\right)+S \operatorname{Pr}_{i}^{\mathcal{W}}(v)}{2}=\operatorname{SPr}_{i}^{\mathcal{W}}(e)
$$

\section{Bilateral probabilistic model}

Consider the following bilateral probabilistic model that supports efficient semiproportional values. Suppose that the player set $N$ is to be randomly partitioned into two nonempty coalitions $S$ and $N \backslash S$ and that from each of these two coalitions a "leader" or "representative" is to be chosen. These two leaders are then to bargain with each other over how to split between the two of them the total resources $v(N)$ available to the $n$ players. Assume that a certain two-person game is chosen, and the two leaders split the amount of $v(N)$ playing the chosen two-person game and having in mind the worths $v(S)$ and $v(N \backslash S)$ of the two coalitions involved. Then after each one of them pays, out of his share, a wage to every member of his coalition as specified by some a priori given wage vector $x \in \mathbb{R}^{n}$, and the rest keeps himself. A payoff vector $x \in \mathbb{R}^{n}$ is consistent (with respect to the probabilistic model) if it coincides with the expected payoff or, which is the same, form a fix point of the described probabilistic procedure.

The case of standard solution for two-person games for uniform distributions of the partition of the player set $N$ and of the choice of a coalition leader was studied in Evans [3]. He showed that the only consistent payoff vector under the circumstances is the Shapley value, and therefore he obtained one more characterization of the Shapley value by means of the described model. Later this result was extended by Driessen and Paulusma [2] for arbitrary probability distributions but within the same standard solution for two-person games framework. Our purpose is to study this probabilistic model for the case of proportional solution for two-person games.

Let $p(S) \geq 0$ denote a probability that a nonempty proper coalition $S$ is to be chosen to form a partition $(S, N \backslash S)$ and $p_{i}^{S} \geq 0$ a probability that a player $i \in S$ will be a leader of $S$, where $S \subset N, S \neq N, S \neq \emptyset$, i.e.,

$$
\sum_{\substack{S \subset N \\ S \neq N \\ S \neq \emptyset}} p(S)=1,
$$

and

$$
\sum_{i \in S} p_{i}^{S}=1, \quad \text { for all } S \subset N, S \neq N, S \neq \emptyset
$$


Hence, $p_{i}=\sum_{\substack{S \subset N \\ S \neq N \\ S \ni i}} p(S) p_{i}^{S}$ is a probability of a player $i$ to be a leader. Moreover, we suppose that the choice of both complementary nonempty proper coalitions $S$ and $N \backslash S$ is equally likely, i.e.,

$$
p(S)=p(N \backslash S), \quad \text { for all } S \subset N, S \neq N, S \neq \emptyset,
$$

or, in other words, that the formation of the partition $(S, N \backslash S)$ is equally likely when any one of two complementary coalitions $S$ and $N \backslash S$ is chosen. In this probabilistic framework, the expected payoff to player $i$ is equal to

$$
E_{i}(v ; x)=\sum_{\substack{S \subset N \\ S \neq N \\ S \ni i}} 2 p(S)\left[\left(1-p_{i}^{S}\right) x_{i}+p_{i}^{S}\left[\frac{v(S)}{v(S)+v(N \backslash S)} v(N)-\sum_{j \in S \backslash i} x_{j}\right]\right] .
$$

A payoff vector $x \in \mathbb{R}^{n}$ is consistent with the probabilistic model if it coincides with the expected payoff, i.e., for all $i \in N, x_{i}=E_{i}(v ; x)$.

Further, following to [2] which results we rely on, we also suppose some weak symmetry condition that any fixed player can be chosen a leader among all coalitions containing his certain fixed partner equally likely for all of his possible partners, i.e., for all $i \in N$,

$$
\sum_{\substack{S \subset N \\ S \neq N \\ S \subseteq\{i, j\}}} p(S) p_{i}^{S} \quad \text { is constant for all } j \in N \backslash i .
$$

Theorem 4.1. Suppose the probability distributions $\{p(S)\}_{\substack{S \subset N \\ S \neq N \\ S \neq \emptyset}},\left\{p_{i}^{S}\right\}_{\substack{S \subset N \\ S \neq N \\ i \in S}}$ satisfy (10) and (11). Then, for every game $v \in \mathcal{G}_{N}^{+}$, there exists a unique efficient payoff vector consistent with the probabilistic model under the proportional solution for two-person games, and it is given by the semiproportional value defined by the collection $\mathcal{W}_{N}$ of weights such that

$$
\gamma_{N}(S ; i)=(n-1) p(S) p_{i}^{S}, \quad \text { for all } S \subset N, S \neq N \text {, and } i \in S,
$$

and

$$
\gamma_{N}(N ; i)=1-(n-1) p_{i}, \quad \text { for all } i \in N .
$$

Remark 4.2. It is worth mentioning that under the collection of weights (12) and (13), the associated semiproportional value satisfies the sufficient efficiency conditions of Proposition 3.4.

When the choice of a size of any nonempty proper coalition is equally likely, and the choice of any nonempty proper coalition among those of the same size is also equally likely, and, moreover, the choice of a leader among all players of any nonempty proper coalition is equally likely as well, the probability distributions $\{p(S)\}_{\substack{S \subset N \\ S \neq N \\ S \neq \emptyset}}$ and $\left\{p_{i}^{S}\right\}_{\substack{S \subset N \\ S \neq N \\ i \in S}}$ are considered to be uniform. In other words, the 
distributions $\{p(S)\} \underset{\substack{S \subset N \\ S \neq N \\ S \neq 0}}{\text { and }}\left\{p_{i}^{S}\right\} \underset{\substack{S \subset N \\ S \neq N \\ i \in S}}{i \in S}$ are uniform if, for all coalitions $S \subset N$,

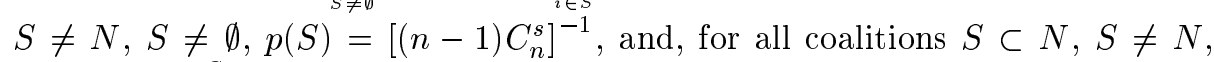
and $i \in S, p_{i}^{S}=1 / s$. It is not difficult to see that for uniform distributions of $p(S)$ and $p_{i}^{S}$ the conditions (10) - (11) are true, and the semiproportional value presenting the unique efficient payoff vector consistent with the probabilistic model under the proportional solution for two-person games coincides with the Shapley type semiproportional value.

If the choice of any nonempty proper coalition is equally likely, and the choice of a leader among all players of any nonempty proper coalition is also equally likely, i.e. if the symmetric probability distributions $p(S)=\frac{1}{2^{n}-2}$, for all coalitions $S \subset N, S \neq N, S \neq \emptyset$, and, for all coalitions $S \subset N, S \neq N$, and $i \in S, p_{i}^{S}=1 / s$ apply, then the conditions (10) - (11) are satisfied, and the semiproportional value of the case (iii) of Example 3.7 arises.

Notice that the Banzhaf type semiproportional value of Example 3.7, being a non-effective value, is not supported by the considered probabilistic model.

Replace now the symmetry condition (11) by a stronger one assuming that for the underlying probability distributions the probability of any player's leadership within a nonempty proper coalition (with reference to the corresponding partition of the player set) is the same for both the coalition and its complementary one enlarged with the given player, i.e., for every $i \in N$, for all $S \subset N$, $S \neq N, S \ni i,|S|>1$,

$$
p(S) p_{i}^{S}=p((N \backslash S) \cup i) p_{i}^{(N \backslash S) \cup i},
$$

and

$$
p(i)=\frac{1}{n-1}-p_{i}
$$

(It is easy to check that symmetry conditions (14) - (15) are stronger than (11), i.e., under (14) - (15), (11) is true.)

Theorem 4.3. Suppose the probability distributions $\{p(S)\}_{\substack{S \subset N \\ S \neq N \\ S \neq 0}},\left\{p_{i}^{S}\right\}_{\substack{S \subset N \\ i \neq N \\ i \in S}}$ meet (10) and (14) - (15). Then the semiproportional value presenting the unique efficient payoff vector consistent with the probabilistic model under the proportional solution for two-person games is proportionally marginalist and for every game $v \in \mathcal{G}_{N}^{+}$, it presents a probabilistic value with respect to the constant-sum normalization game $v^{c}$.

Remark 4.4. Observe, that the semiproportional value defined by the collection of weights (12) - (13) under assumptions (10) and (14) - (15) gives an example of efficient and probabilistic value (with respect to constant-sum normalization game) that is not necessary the random-order one (compare to Theorem 13 in Weber [12]). This happens because the set of games $\mathcal{G}_{N}^{+c}$ does not contain the full class of unanimity games.

The Shapley type semiproportional value of Example 3.7 is supported by the considered probabilistic model under the proportional solution for two-person 
games when underlying probability distributions satisfy the conditions (10) and (14) - (15). It turns out that the Shapley type semiproportional value can be characterized by efficiency, symmetry, and proportional marginalism, similarly to Young's axiomatization [13] for the Shapley value via efficiency, symmetry, and marginalism. Notice that independence of these three conditions of efficiency, symmetry, and proportional marginalism one can get almost directly from Young.

Theorem 4.5. The only efficient, symmetric, and proportionally marginalist value defined on $\mathcal{G}_{N}^{+}$is the Shapley type semiproportional value.

Remark 4.6. It should be noted that on $\mathcal{G}_{N}^{+}$efficiency, symmetry, and proportional marginalism guarantee the null player property similarly as on $\mathcal{G}_{N}$ the null-player property follows from efficiency, symmetry, and marginalism. (The proof is given in Appendix).

It follows directly from Theorems 4.3 and 4.5 that for all probability distributions meeting the assumptions (10) and (14) - (15) there exists the only one symmetric semiproportional value presenting the unique efficient payoff vector consistent with the relevant probabilistic model under the proportional solution for two-person games, and it coincides with the symmetric and proportionally marginalist Shapley type semiproportional value.

Theorem 4.7. For every game $v \in \mathcal{G}_{N}^{+}$, the only efficient and symmetric payoff vector consistent with the probabilistic model under the proportional solution for two-person games and assumptions (10), (14) - (15) concerning the probability distributions $\{p(S)\}_{\substack{S \subset N \\ S \neq N \\ S \neq \emptyset}},\left\{p_{i}^{S}\right\}_{\substack{S \subset N \\ S \neq N \\ S \ni i}}$, is the Shapley type semiproportional value.

Therefore, Theorem 4.7 provides another characterization of the Shapley type semiproportional value by means of the family of probabilistic models under the proportional solution for two-person games for which underlying probability distributions meet the conditions (10) and (14) - (15).

\section{Appendix: Proofs}

Proof of Proposition 3.3. For any two-person game $v \in \mathcal{G}_{\{1,2\}}^{+}$, for every $i, j \in$ $\{1,2\}, j \neq i$, by Definition 3.1,

$$
\operatorname{SPr}_{i}^{\mathcal{W}}(v) \stackrel{(4)}{=} \gamma_{\{1,2\}}(\{i\}, i) \frac{v(i)-v(j)}{v(i)+v(j)} v(1,2)+\gamma_{\{1,2\}}(\{1,2\}, i) v(1,2) .
$$

Because of the proportionality rule, as given by (3), we have:

$$
\operatorname{SPr}_{i}^{\mathcal{W}}(v)=\frac{v(i)}{v(1)+v(2)} v(1,2) .
$$


Hence,

$$
\gamma_{\{1,2\}}(\{i\}, i)[v(i)-v(j)]+\gamma_{\{1,2\}}(\{1,2\}, i)[v(i)+v(j)]=v(i),
$$

i.e.,

$$
\begin{aligned}
& {\left[\gamma_{\{1,2\}}(\{i\}, i)+\gamma_{\{1,2\}}(\{1,2\}, i)-1\right] v(i)+} \\
& +\left[\gamma_{\{1,2\}}(\{1,2\}, i)-\gamma_{\{1,2\}}(\{i\}, i)\right] v(j)=0 .
\end{aligned}
$$

Therefore, since the game $v$ can be chosen arbitrary, for every $i=1,2$,

$$
\gamma_{\{1,2\}}(\{i\}, i)+\gamma_{\{1,2\}}(\{1,2\}, i)=1 \quad \text { and } \quad \gamma_{\{1,2\}}(\{1,2\}, i)-\gamma_{\{1,2\}}(\{i\}, i)=0 \text {. }
$$

From where, for every $i=1,2$,

$$
\gamma_{\{1,2\}}(\{i\}, i)=\gamma_{\{1,2\}}(\{1,2\}, i)=\frac{1}{2} .
$$

Proof of Proposition 3.4. Straightforward computations yield the following chain of equalities:

$$
\begin{aligned}
& \sum_{i=1}^{n} \operatorname{SPr}_{i}^{\mathcal{W}}(v) \stackrel{(5)}{=} \sum_{i=1}^{n} \sum_{\substack{\subseteq \subseteq N \\
S \ni i}} \gamma_{N}(S, i)\left[v^{c}(S)-v^{c}(N \backslash S)\right]= \\
& =\sum_{\substack{S \subseteq N \\
S \neq \emptyset}} \sum_{i \in S} \gamma_{N}(S, i)\left[v^{c}(S)-v^{c}(N \backslash S)\right]= \\
& =\sum_{\substack{S \subset N \\
S \neq \emptyset}}\left[v^{c}(S)-v^{c}(N \backslash S)\right] \sum_{i \in S} \gamma_{N}(S, i)= \\
& =v^{c}(N) \sum_{i \in N} \gamma_{N}(N, i)+\frac{1}{2}\left[\sum_{\substack{S \subset N \\
S \neq N \\
S \neq \emptyset}}\left[v^{c}(S)-v^{c}(N \backslash S)\right] \sum_{i \in S} \gamma_{N}(S, i)+\right. \\
& \left.+\sum_{\substack{S \subset N \\
S \neq N \\
S \neq \emptyset}}\left[v^{c}(N \backslash S)-v^{c}(S)\right] \sum_{i \in N \backslash S} \gamma_{N}(N \backslash S, i)\right]= \\
& \stackrel{(6)}{=} v(N)+\frac{1}{2}\left[\sum_{\substack{S \subset N \\
S \neq N \\
S \neq \emptyset}}\left[v^{c}(S)-v^{c}(N \backslash S)\right]\right] \times \\
& \times\left[\sum_{i \in S} \gamma_{N}(S, i)-\sum_{i \in N \backslash S} \gamma_{N}(N \backslash S, i)\right]= \\
& \stackrel{(7)}{=} v(N) \text {. }
\end{aligned}
$$


Proof of Proposition 3.5. For every $i \in N$ by straightforward computations we can obtain:

$$
\begin{aligned}
& \operatorname{SPr}_{i}^{\mathcal{\mathcal { W }}}(v) \stackrel{(5)}{=} \sum_{\substack{S \subset N \\
S \ni i}} \gamma_{N}(S, i)\left[v^{c}(S)-v^{c}(N \backslash S)\right]= \\
& \stackrel{(8)}{=} \frac{1}{2} \sum_{\substack{S \subset N \\
S \ni i}} \gamma_{N}(S, i)\left[\left[v^{c}(S)-v^{c}(N \backslash S)\right]+\left[v^{c}((N \backslash S) \cup i)-v^{c}(S \backslash i)\right]\right]= \\
& =\frac{1}{2} \sum_{\substack{S \subseteq N \\
S \ni i}} \gamma_{N}(S, i)\left[\left[v^{c}(S)-v^{c}(S \backslash i)\right]+\left[v^{c}((N \backslash S) \cup i)-v^{c}(N \backslash S)\right]\right]= \\
& \stackrel{(8)}{=} \sum_{S \subseteq N \backslash i} \gamma_{N}(S \cup i, i)\left[v^{c}(S \cup i)-v^{c}(S)\right] .
\end{aligned}
$$

Proof of Proposition 3.8. By [5], for any game $v$, the Shapley value $S h(v)$ solves

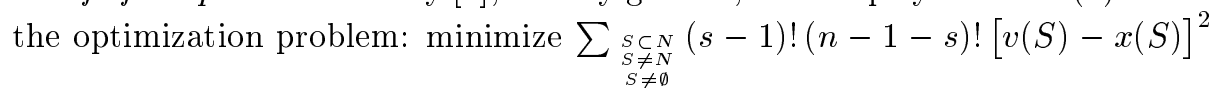
subject to $x(N)=v(N)$. From this, together with the relationship $\operatorname{SPr}^{S h}(v)=$ $S h\left(N, v^{c}\right)$, we deduce that $S \operatorname{Pr}^{S h}(v)$ solves the following optimization problem: $\operatorname{minimize} \sum_{\substack{s \subset N \\ S \neq N}}(s-1) !(n-1-s) !\left[v^{c}(S)-x(S)\right]^{2}$ subject to $x(N)=v(N)$. For all $S \subset N, S \neq N, S \neq \emptyset$, straightforward computations yield, the following chain of equalities (subject to $x(N)=v(N)$ ) that completes the proof:

$$
\begin{aligned}
v^{c}(S)-x(S) & =\left[\frac{v(S)}{v(S)+v(N \backslash S)}\right] v(N)-x(S)= \\
& =\frac{v(S) x(N)-x(S)[v(S)+v(N \backslash S)]}{v(S)+v(N \backslash S)}= \\
& =\frac{v(S) x(N \backslash S)-x(S) v(N \backslash S)}{v(S)+v(N \backslash S)}= \\
& =\frac{\frac{x(N \backslash S)}{v(N \backslash S)}-\frac{x(S)}{v(S)}}{\frac{1}{v(N \backslash S)}+\frac{1}{v(S)}}
\end{aligned}
$$

Proof of Proposition 3.9. Next chain of equalities proves the first statement and the second one is a direct consequence of the first. 
For all positive games $v \in \mathcal{G}_{N}^{+}$, for every $i \in N$,

$$
\begin{aligned}
& \operatorname{SPr}_{i}^{\mathcal{W}}\left(\frac{1}{v}\right) \stackrel{(4)}{=} \gamma_{N}(N, i) \frac{1}{v}(N)+\sum_{\substack{S \subset N \\
S \neq N \\
S \ni i}} \gamma_{N}(S, i)\left[\frac{\frac{1}{v}(S)-\frac{1}{v}(N \backslash S)}{\frac{1}{v}(S)+\frac{1}{v}(N \backslash S)}\right] \frac{1}{v}(N)= \\
& =\frac{\gamma_{N}(N, i)}{v(N)}+\sum_{\substack{S \subset N \\
S \neq N \\
S \ni i}} \gamma_{N}(S, i)\left[\frac{\frac{1}{v(S)}-\frac{1}{v(N \backslash S)}}{\frac{1}{v(S)}+\frac{1}{v(N \backslash S)}}\right] \frac{1}{v(N)}= \\
& =\frac{\gamma_{N}(N, i)}{v(N)}+\sum_{\substack{S \subset N \\
S \neq N \\
S \ni i}} \gamma_{N}(S, i)\left[\frac{v(N \backslash S)-v(S)}{v(N \backslash S)+v(S)}\right] \frac{1}{v(N)}= \\
& =\frac{\gamma_{N}(N, i)}{v(N)}-\frac{1}{(v(N))^{2}} \sum_{\substack{S \subset N \\
S \neq N \\
S \ni i}} \gamma_{N}(S, i)\left[\frac{v(S)-v(N \backslash S)}{v(S)+v(N \backslash S)}\right] v(N)= \\
& \stackrel{(4)}{=} \frac{\gamma_{N}(N, i)}{v(N)}-\frac{1}{(v(N))^{2}}\left[\operatorname{SPr}_{i}^{\mathcal{W}}(v)-\gamma_{N}(N, i) v(N)\right]= \\
& =-\frac{\operatorname{SPr}_{i}^{\mathcal{W}}(v)}{(v(N))^{2}}+2 \frac{\gamma_{N}(N, i)}{v(N)}
\end{aligned}
$$

Proof of Theorem 4.1. For any two complementary coalitions $S$ and $N \backslash S$, the proportionality rule (3) applied to their two-person "subgame" of any game $v \in \mathcal{G}_{N}^{+}$agrees with the standard solution to their two-person "subgame" of the constant-sum normalization $v^{c}$. Therefore, the validity of the theorem follows almost directly from Theorem 2.1 in [2] that states that, for every game $v \in \mathcal{G}_{N}^{+}$ and, in particular, $v^{\prime}=v^{c}$ under some $v \in \mathcal{G}_{N}^{+}$, the only efficient payoff vector consistent with the probabilistic model under the standard solution for twoperson games is given, for all $i \in N$, by the formula

$$
x_{i}=\left[1-(n-1) p_{i}\right] v^{c}(N)+(n-1) \sum_{\substack{S \subset N \\ S \neq N \\ S \ni i}} p(S) p_{i}^{S}\left[v^{c}(S)-v^{c}(N \backslash S)\right] .
$$

Returning back from $v^{c}$ to $v$, we obtain $x_{i}=\operatorname{SPr}_{i}^{\mathcal{W}}(v)$ with the collection of weights defined by (12) - (13).

Proof of Theorem 4.3. It is not difficult to see that under assumptions (14) - (15) the semiproportional value defined by the collection of weights (12) - (13) satisfies conditions of Proposition 3.5. Moreover, all these weights are non-negative and weights underlying any player's payoff represent a probability distribution for all coalitions containing the player. 
Proof of Theorem 4.5. It was already noticed that the proportional marginalism of the value defined on $\mathcal{G}_{N}^{+}$is equivalent to marginalism of this value considered on $\mathcal{G}_{N}^{+c}$, and the Shapley type semiproportional value defined on $\mathcal{G}_{N}^{+}$and restricted to $\mathcal{G}_{N}^{+c}$ coincides with the Shapley value on $\mathcal{G}_{N}^{+c}$. To complete the proof it suffices to refer to Theorem 1 in [6].

Proof of Remark 4.6. ${ }^{1}$ As it was mentioned above it is enough to show that every efficient, symmetric, and marginalist value $\xi$ defined on $\mathcal{G}_{N}^{+c}$ possesses the null-player property. Consider a symmetric game $\hat{v} \in \mathcal{G}_{N}^{+c}$ defined as

$$
\hat{v}(S)=s, \quad \text { for all } S \subseteq N .
$$

Since $\xi$ is efficient and symmetric, for all $i \in N$,

$$
\xi_{i}(\hat{v})=1 .
$$

Because of marginalism, for every $i \in N, \xi_{i}$ depends only on $i$ th vector of marginal utilities. For all $S \subseteq N \backslash i$,

$$
\hat{v}(S \cup i)-\hat{v}(S)=1 .
$$

Hence, for $\xi$ presented in the marginalist form (1) we have,

$$
\phi_{i}(1, \ldots, 1)=1, \quad \text { for all } i \in N .
$$

Now, we fix some player $i \in N$ and consider the unanimity game $u_{i}$, i.e.,

$$
u_{i}(S)=\left\{\begin{array}{ll}
1, & S \ni i, \\
0, & S \not \nexists i,
\end{array} \quad \text { for all } S \subseteq N .\right.
$$

It is not difficult to see that $u_{i} \in \mathcal{G}_{N}^{+c}$ and, for all $S \subseteq N \backslash i$,

$$
u_{i}(S \cup i)-u_{i}(S)=1 .
$$

By assumption, the value $\xi$ is marginalist and, therefore, from (16) we have that

$$
\xi_{i}\left(u_{i}\right)=1 .
$$

Because of efficiency and symmetry, in the game $u_{i}$ all other players $j \in N$, $j \neq i$, get nothing, i.e.,

$$
\xi_{j}\left(u_{i}\right)=0, \quad \text { for all } j \in N \backslash i \text {. }
$$

But in the game $u_{i}$ all players $j \in N, j \neq i$, are null-players and, therefore, for $\xi$ presented in the marginalist form (1), we have

$$
\phi_{j}(0, \ldots, 0)=0, \quad \text { for all } j \in N \backslash i .
$$

From here, because of symmetry, it follows that $\xi$ possesses the null-player property.

\footnotetext{
${ }^{1}$ The proof of the null-player property is due to Elena Yanovskaya.
} 


\section{References}

1. Banzhaf JF (1965) Weighted voting doesn't work: a mathematical analysis. Rutgers Law Review 19:317-343

2. Driessen TSH, Paulusma D (2001) Two extensions of the Shapley value for cooperative games. Mathematical Methods of Operation Research 53(1):35-49

3. Evans RA (1996) Value, consistency, and random coalition formation. Games and Economic Behavior 12:68-80

4. Feldman B (1999) The proportional value. Discussion paper, Scudder Kemper Investments, Chicago, USA, presented at The X International Game Theory Conference at Stony Brook, USA, July 19-23, 1999

5. Keane M (1965) Some Topics in N-Person Game Theory. Ph.D. thesis, Northwestern University, Evanston, Illinois

6. Khmelnitskaya AB (2002) Shapley value for constant-sum games. Memorandum No. ..., Faculty of Mathematical Sciences, University of Twente, Enschede, The Netherlands

7. Naumova NI (2000) Weighted entropy solution for cooperative games. In: Control Applications of Optimization 11 IFAC International Workshop at St.Petersburg, Russia, July 3-6, 2000, Abstracts, pp. 169-170

8. Ortmann KM (2000) The proportional value for positive cooperative games. Mathematical Methods of Operations Research 51:235-248

9. Rothblum UG (1988) Combinatorial representations of the Shapley value based on average relative payoffs. In: Roth A (ed.) The Shapley value: Essays in honor of Lloyd S. Shapley. Cambridge University Press, Cambridge, UK, pp. 121-126

10. Shapley LS (1953) A value for $n$-person games. In: Tucker AW, Kuhn HW (eds.) Contributions to the theory of games II. Princeton University Press, Princeton, NJ, pp. 307-317

11. Vorob'ev NN, Liapunov AN (1998) The proper Shapley value. In: Petrosjan LA, Mazalov VV (eds.) Game Theory and Applications IV. Nova Science Publishers, pp. $155-159$

12. Weber RG (1988) Probabilistic Values for Games. In: Roth A (ed.) The Shapley Value: Essays in Honor of Lloyd S. Shapley. Cambridge University Press, Cambridge, UK, 1988, pp. 101-119

13. Young HP (1985) Monotonic solutions of cooperative games. International Journal of Game Theory 14:65-72 